\title{
Influencia de la minería en las características fisicoquímicas en la parte media y baja del río Quito, Chocó, Colombia
}

\section{Influence of mining in the physicochemical characteristics in its middle and low part the Quito river, Chocó, Colombia}

\author{
Yirlesa Murillo Hinestroza ${ }^{1}$, Lady Vargas Porras ${ }^{2}$
}

\section{Resumen}

El río Quito se constituye en la corriente principal que surca el territorio del municipio del mismo nombre, el cual tiene un caudal en verano de $200 \mathrm{~m}^{3} / \mathrm{seg}$ haciéndolo navegable durante todo el año. Sobre este y algunos de sus afluentes, se desarrollan actividades mineras. Con la finalidad de analizar los aportes contaminantes de fuentes hídricas que vierten al río Quito, se realizaron mediciones, in situ y de laboratorio, de variables fisicoquímicas como temperatura, conductividad, sólidos disueltos, sólidos suspendidos, turbiedad, oxígeno disuelto, $\mathrm{pH}$, hierro, nitrato, nitrito, sulfato, fosfato, sólidos totales, grasas y aceites, tanto en la parte media como baja de la cuenca teniendo en cuenta algunos confluencias de otros cuerpos de agua a la misma. Se seleccionaron seis puntos de muestreo en la parte media y cuatro en la parte baja, para un total de diez puntos; a partir del análisis realizado en los tramos muestreados, queda al descubierto que el desarrollo de actividades mineras sobre el río Quito y otros cuerpos de agua que a este vierten, representa una alta influencia en las características fisicoquímicas del agua y por lo tanto en la calidad de la misma sobre todo en su parte media; todo esto teniendo en cuenta, que se incrementaron los valores de solidos disueltos, suspendidos y totales, al igual que la turbiedad del agua y otras variables fisicoquimicas como algunos nutrientes junto con las grasas y aceites.

Palabras clave: Actividades mineras, Calidad del agua, Conservación, Ecosistemas, Parámetros fisicoquímicos, Río Quito.

\section{Abstract}

The Quito river is the main stream that runs through the territory of the municipality of the same name, which has a summer flow of $200 \mathrm{~m}^{3} / \mathrm{sec}$ making it navigable throughout the year. On this
Ingeniera Ambiental, Instituto de Investigaciones Ambientales del Pacífico (IIAP), Quibdó, Chocó, Colombia. e-mail: yirdavid@hotmail.com

2 Ingeniera Ambiental y Sanitaria, Especialista en Gestión del Recurso Hídrico, Ms (C) Ciencias Ambientales, Instituto de Investigaciones Ambientales del Pacífico(IIAP), Quibdó, Chocó, Colombia. e-mail: 1ady8181@gmail.com 


\section{Bioetnia Volumen 12, 2015}

and some of its tributaries, mining activities are developed. In situ and laboratory measurements of physicochemical variables such as temperature, conductivity, dissolved solids, suspended solids, turbidity, dissolved oxygen, $\mathrm{pH}$, iron, nitrate, nitrite, sulfate, phosphate, total solids, fats and oils were performed in both the average as low of the basin taking into account some confluences of other bodies of water to it. This is done with the purpose of analyzing the contaminant inputs from water sources that flow into the Quito river. Six sampling points were selected in the middle and four in the lower part, for a total of ten sampling points; from the analysis carried out on the sampled sections, it is revealed that the development of mining activities on the Quito river and other bodies of water that discharge it represents a high influence on the physicochemical characteristics of the water and therefore on the quality of the same especially in the middle part. The above taking into account that the values of solids dissolved, suspended and total, as well as turbidity of water and other physicochemical variables such as some nutrients together with fats and oils.

Keywords: Conservation, Ecosystems, Mining activities, Physicochemical parameters, Quito River, Water quality.

\section{Introducción}

Las fuentes hídricas y en especial los ríos, no solo constituyen un elemento vital para el desarrollo de la vida de los seres humanos, sino también para las comunidades biológicas en su interior, para los cuales son un ecosistema que incluye diferentes tipos de hábitat que les permite desarrollar todos sus procesos vitales. De modo similar las comunidades localizadas en el área de influencia del río Quito, ven en este un ecosistema que trae consigo multiplicidad de bienes y servicios, de ahí que la fuente hídrica sea utilizada como medio de sustento, desarrollo y en especial su principal medio de transporte. A este respecto Millenium Ecosystems Assesment (2005) manifiesta que hay una estrecha relación entre los ecosistemas acuáticos y las poblaciones humanas, porque ambos se benefician de muchos bienes y servicios ofrecidos por estos, dentro de los cuales se destacan la producción de comida (pesca), madera, el turismo, la recreación y el transporte.

Sin embargo, el desarrollo de actividades mineras realizadas sobre las márgenes del río y sus afluentes, traen consigo notables afectaciones en las características fisicoquímicas del agua, por lo que se han generado impactos sobre el recurso, razón por la cual el río Quito presenta notables afectaciones sobre su estructura y funcionamiento ecosistémico, debido a que el desarrollo de actividades mineras realizadas con dragas y retroexcavadoras, que remueven del fondo del cauce y de las riberas toneladas de material que es filtrado para obtener metales, ha ocasionado no solo una modificación de los cauces, sino también importantes cambios en el balance de agua entre infiltración y escorrentía, debido a la modificación del suelo, la eliminación de la cobertura vegetal aumentando la capacidad erosiva. En relación con esto Clark (1995) expresa que la realización de actividades mineras produce una alteración del cauce de los ríos, al igual que impactos dramáticos tanto en la dinámica e hidrología de los mismos como en las comunidades bióticas asociadas. Además, la grave alteración del cauce que produce el dragado, también afecta seriamente los lugares de alimentación, refugio y reproducción de muchas especies acuáticas, incluyendo peces, quelonios acuáticos, moluscos y crustáceos, así como de los invertebrados que les sirven de alimento.

Teniendo en cuenta lo expuesto, se evaluó la calidad del agua del río Quito mediante la medición y análisis de variables fisicoquímicas que ponen al descubierto el estado del recurso y que además podrían generar instrumentos de gestión y control del uso y manejo del ecosistema, lo que a su vez garantiza la conservación de organismos acuáti- 
cos, el mantenimiento del equilibrio ecológico al interior de estos ecosistemas y el mejoramiento de la calidad de vida de los asentamientos humanos en su área de influencia.

Se presentan entonces los resultados de este análisis como un aporte al conocimiento del estado de la cuenca del río Quito, información que sirve como base para el diseño de medidas encaminadas a la prevención y mitigación de los impactos causados directa e indirectamente sobre la fuente en atención al estado de la intervención minera actual.

Área de estudio. La cuenca hidrográfica del río Quito, se encuentra localizada en la parte central del departamento de Chocó, con un área de drenaje de $166.889,04$ hectáreas $\left(1.668,89 \mathrm{~km}^{2}\right)$ y drena sus aguas al río Atrato a la altura de la cabecera municipal del municipio de Quibdó. El área de muestreo abarcó la parte media y baja del río Quito, en los tramos de Villaconto-Paimado y Villaconto-Cunrundo, respectivamente. Estos se localizaron en las coordenadas $5^{\circ} 25^{\prime} \mathrm{N}, 76^{\circ} 40^{\prime}$ W y $5^{\circ} 38^{\prime} 33.9^{\prime \prime} \mathrm{N}, 76^{\circ} 43^{\prime} 18.2 \mathrm{~W}$. En términos generales, estas zonas de muestreo se encuentran dentro de un ecosistema intervenido por actividades mineras realizadas con dragas. Las zonas se caracterizaron por presentar espejos de agua dulce con cauces profundos y aguas turbias, lechos cubiertos de arena y grava, además, de una vegetación discontinua sobre sus márgenes (Figura 1).

\section{Métodos}

Previo a un recorrido por el sistema hídrico, se seleccionaron seis puntos de muestreo en la parte media y cuatro en la parte baja, para un total de diez puntos (Tabla 1, Figura 2). En cada punto se hicieron mediciones in situ de variables fisicoquímicas como temperatura, conductividad, sólidos disueltos, sólidos suspendidos, turbiedad, oxígeno disuelto, $\mathrm{pH}$, hierro, nitrato, nitrito, sulfato, y fosfato, utilizando un colorímetro portátil

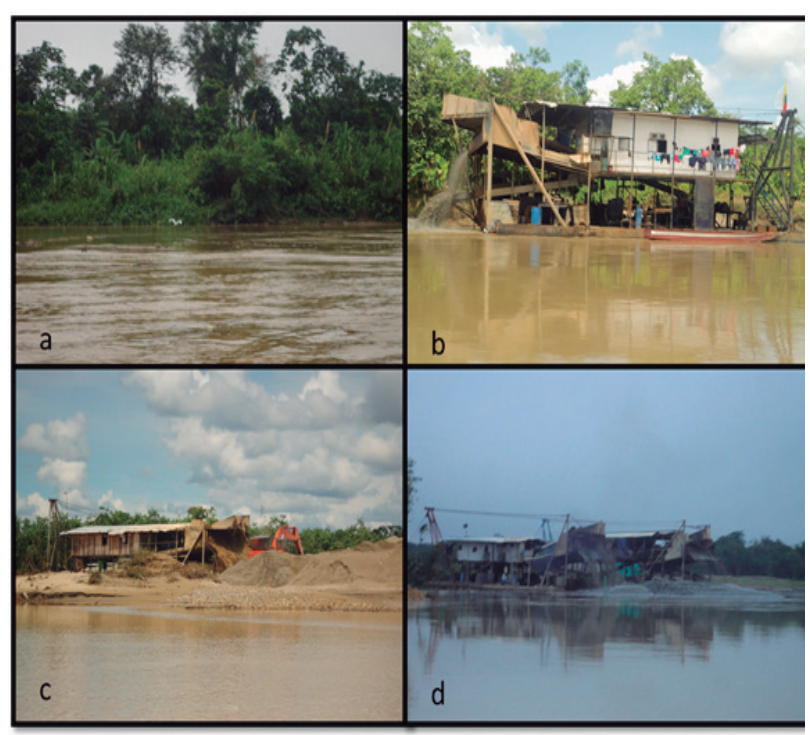

Figura 1. a: Aguas caudalosas; b,c,d: Aguas turbias y actividades mineras sobre el río Quito.

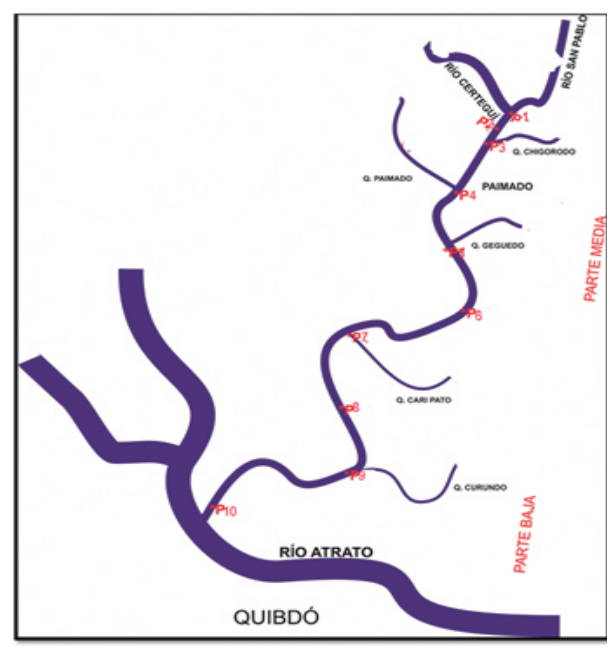

Figura 2. Esquema de distribución de los puntos de muestreo en el área de estudio.

HACH 850, un multiparámetro Ysi Profesional Plus Quick 1700/1725 y un GPS para la georeferenciación de cada punto (Figura 3). Aunado a esto, en los puntos $1,3,4,5,7,9$, se tomaron muestras de agua para análisis en el laboratorio de la Corporación Autónoma Regional del Chocó (CODECHOCO) de variables fisicoquímicas como sólidos totales, grasas y aceites, para lo cual se tuvo en cuenta la ubicación de las dragas $\mathrm{y}$ algunos asentamientos humanos. 
Bioetnia Volumen 12, 2015

Tabla 1. Distribución y localización de los puntos de muestreo

\begin{tabular}{lll}
\hline Punto & \multicolumn{1}{c}{ Descripción } & Coordenadas \\
\hline 1 & Desembocadura de los ríos Cetegui y San Pablo al río Quito & $5^{\circ} 5^{\prime} 144^{\prime \prime}-76^{\circ} 43^{\prime} 20.4^{\prime \prime}$ \\
2 & Agua bajo de la confluencia entre los ríos Certegui y Sampablo al río Quito & $5^{\circ} 25^{\prime} 38.9^{\prime \prime}-76^{\circ} 43^{\prime} 24.5^{\prime \prime}$ \\
3 & Desembocadura de la quebrada Chigorodó al río Quito & $5^{\circ} 27^{\prime} 46.7^{\prime \prime}-76^{\circ} 44^{\prime} 08.2^{\prime \prime}$ \\
4 & Desembocadura de la quebrada Paimado al río Quito & $5^{\circ} 28^{\prime} 18.6^{\prime \prime}-76^{\circ} 43^{\prime} 08.6^{\prime \prime}$ \\
5 & Desembocadura de la quebrada Queguedo al río Quito & $5^{\circ} 30^{\prime} 23.1^{\prime \prime}-76^{\circ} 44^{\prime} 39.4^{\prime \prime}$ \\
6 & Agua debajo de la confluencia entre la quebrada Queguedo al río Quito & $5^{\circ} 27^{\prime} 47.7^{\prime \prime}-76^{\circ} 45^{\prime} 22.8^{\prime \prime}$ \\
7 & Desembocadura de la quebrada Caripato al río Quito & $5^{\circ} 36^{\prime} 33.9^{\prime \prime}-76^{\circ} 44^{\prime} 46.6^{\prime \prime}$ \\
8 & Agua debajo de la confluencia entre la quebrada Caripato al río Quito & $5^{\circ} 38^{\prime} 22.5^{\prime \prime}-76^{\circ} 43^{\prime} 22.5^{\prime \prime}$ \\
9 & Desembocadura de la quebrada Curundo al río Quito & $5^{\circ} 38^{\prime} 28.1^{\prime \prime}-76^{\circ} 43^{\prime} 12.0^{\prime \prime}$ \\
10 & Aguas abajo de la confluencia entre la quebrada Curundó y el río Quito & $5^{\circ} 38^{\prime} 51.7^{\prime \prime}-76^{\circ} 43^{\prime} 06.3^{\prime \prime}$ \\
\hline
\end{tabular}

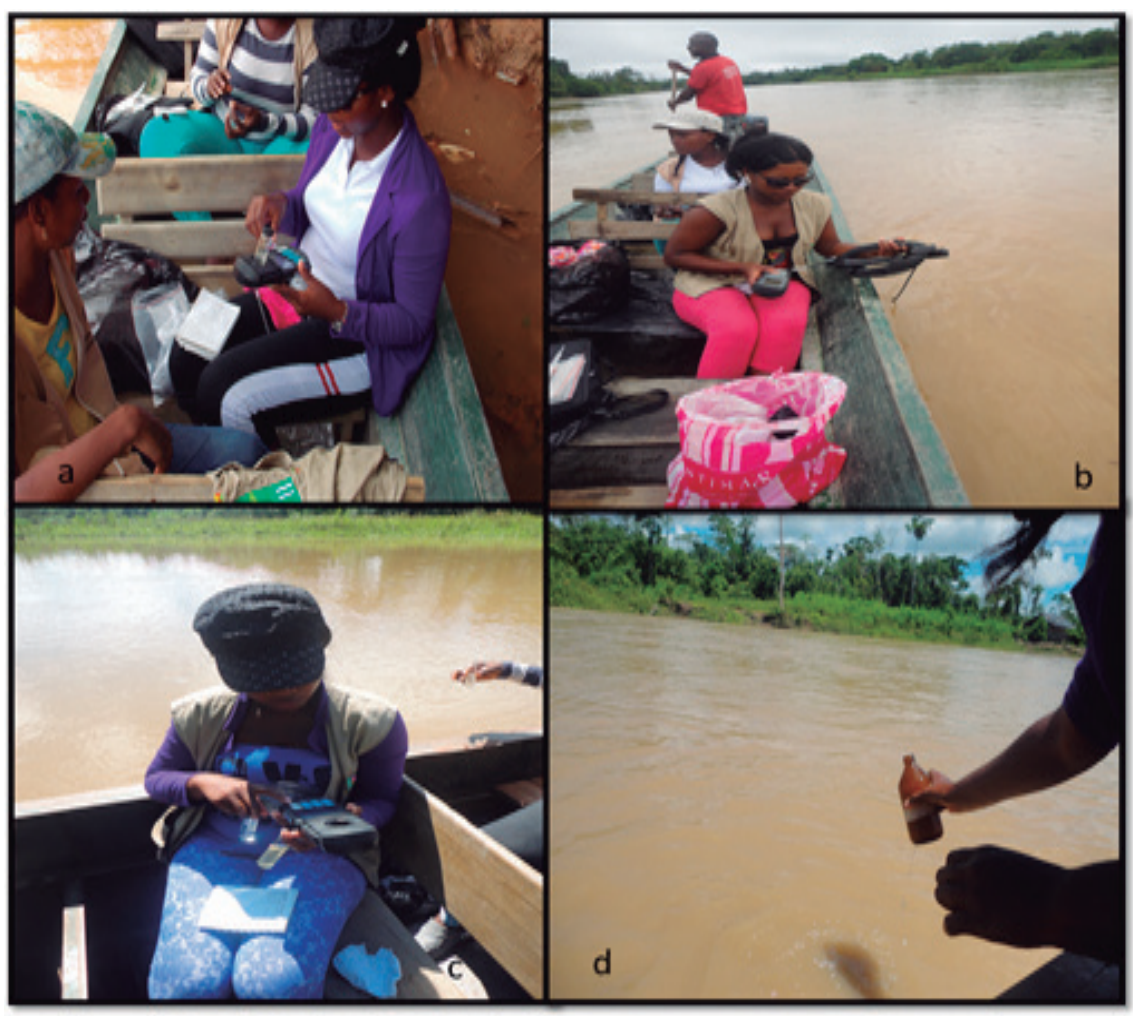

Figura 3. Medición in situ de variables fisicoquímicas en el río Quito.

Estas mediciones in situ se realizaron con la finalidad de analizar los aportes contaminantes de fuentes hídricas que vierten al río Quito. Para analizar el estado y la conservación del agua como ecosistema prioritario para el establecimiento de grupos biológicos al interior del río Quito, se hizo una comparación de los datos obtenidos con los estándares de calidad para la preservación de la fauna y la flora acuática, así como con los datos arrojados por otras investigaciones de calidad de agua en fuentes superficiales intervenidas por actividades mineras. También los resultados se 
relacionaron con las observaciones realizadas en campo sobre el estado del agua, su dinámica de flujo y su interacción con componentes biológicos.

\section{Resultados y discusión}

Análisis de variables fisicoquímicas en la parte media y baja del río Quito. Los resultados obtenidos permitieron determinar que la calidad del agua del río Quito y sus condiciones naturales, se encuentran alteradas y se puede inferir que esta alteración está influida por la presencia de la actividad minera en la zona, pues variables como oxígeno disuelto y $\mathrm{pH}$, que determinan la presencia de vida en el agua, se encuentran en concentraciones óptimas; sin embargo, las mayores alteraciones a nivel fisicoquímico se encuentran en variables como turbiedad, sólidos y algunos iones que están asociados con el aporte de material particulado ejercido por esta actividad productiva que ha redundado en una modificación del sistema hídrico como hábitat de grupos biológicos y fuente de desarrollo de las comunidades aledañas.

La Tabla 2 presentan los resultados obtenidos para las concentraciones de variables fisicoquímicas medidas in situ. Se encontró, que los sólidos suspendidos y la turbiedad, alcanzaron hasta 770 , $601 \mathrm{mg} / \mathrm{l}$ y >1100, 578 FAU tanto en la zona media y baja de la cuenca respectivamente, evidenciándose los mayores valores en la parte media, situación que pudo deberse a la gran cantidad de material granulado que es aportado por los cerca de 11 entables mineros ubicados en este sector, los cuales dentro de su proceso remueven las capas del suelo y captan el agua directamente del río para su lavado y obtención del mineral, vertiendo al cauce el material sobrante sin tratamiento previo.

Además, se pueden explicar los altos valores de turbiedad encontrados en la parte media del río, teniendo en cuenta que en este sector se observó la existencia de otros entables mineros y el vertimiento de cuerpos de agua como los de los ríos Sampablo y Certegui, los cuales aportan gran cantidad de partículas en suspensión (Figura 4). Todo este cúmulo de material aportado

Tabla 2. Valores obtenidos in situ y de laboratorio de variables fisicoquímicas en la parte media y baja del río Quito

\begin{tabular}{|c|c|c|c|c|c|c|c|c|c|c|c|c|}
\hline & \multicolumn{7}{|c|}{ Parte media del río Quito (punto) } & \multicolumn{5}{|c|}{ Parte baja del río Quito (punto) } \\
\hline & 1 & 2 & 3 & 4 & 5 & $6 P$ & Promedio & 7 & 8 & 9 & $10 P$ & Promedio \\
\hline Temperatura $\left({ }^{\circ} \mathrm{C}\right)$ & 26,3 & 26,3 & 27,5 & 26,8 & 26,4 & 26,7 & 26,6 & 26,7 & 27,0 & 26,5 & 27,2 & 26,8 \\
\hline Oxígeno disuelto (mg/l) & 6,8 & 6,9 & 6,1 & 6,3 & 6,2 & 7,3 & 6,6 & 5,7 & 5,5 & 5,3 & 6,2 & 5,6 \\
\hline Conductividad ( $\mu \mathrm{s} / \mathrm{cm})$ & 10,6 & 11,7 & 10,1 & 8,3 & 9,1 & 10,0 & 9,9 & 19,2 & 15,23 & 17,56 & 17,15 & 17,2 \\
\hline Sólidos disueltos Totales TSD (mg/l) & ) 424 & 7,16 & 232 & 80 & 84 & 6,5 & 138,9 & 220 & 9,53 & 156 & 1,72 & 96,8 \\
\hline $\mathrm{PH}$ & 5,9 & 6,0 & 5,4 & 5,0 & 5,5 & 5,2 & 5,5 & 5,9 & 5,8 & 6,0 & 6,0 & 5,9 \\
\hline Nitratos (mg/l) & 7,50 & 23,15 & 1,52 & 3,74 & 2,26 & 1,83 & 6,6 & 25,43 & 20,05 & 24,5 & $>35,0$ & 26,2 \\
\hline Turbiedad (FAU) & 392 & 123 & $>1100$ & 330 & 172 & $>1100$ & $0 \quad 536,1$ & 250 & 555 & 303 & 578 & 421,5 \\
\hline Sólidos suspendidos (mg/l) & 770 & 126 & 712 & 38 & 78 & 530 & 375,6 & 224 & 601 & 270 & 601 & 424 \\
\hline Nitritos (mg/l) & 0,986 & 0,238 & 1,175 & 0,660 & 0,439 & 1,140 & 0,773 & 0,59 & 0,98 & 0,514 & 1,056 & 0,785 \\
\hline Sulfato (mg/l) & 60 & 18 & $>80$ & 55 & 19 & $>80$ & 52 & 49 & 69 & 57 & 74 & 62,2 \\
\hline Hierro $(\mathrm{mg} / \mathrm{l})$ & $>3,30$ & 2,23 & $>3,30$ & 2,44 & $>3,30$ & $>3,30$ & $0 \quad 2,97$ & 2,99 & $>3,30$ & 2,72 & $>3,30$ & 3,07 \\
\hline Fosfato (mg/l) & 2,67 & 1,03 & $>2,75$ & 1,41 & 1,32 & $>2,75$ & $5 \quad 1,98$ & 1,62 & 2,73 & 1,93 & $>2,75$ & 2,25 \\
\hline Sólidos totales (mg/l) & 952 & & 728 & 116 & 140 & & 484 & 296 & & 308 & & 302 \\
\hline Grasas y aceites (mg/l) & 27 & & 46,5 & 48 & 45 & & 27,75 & 39 & & 18,5 & & 28,7 \\
\hline
\end{tabular}




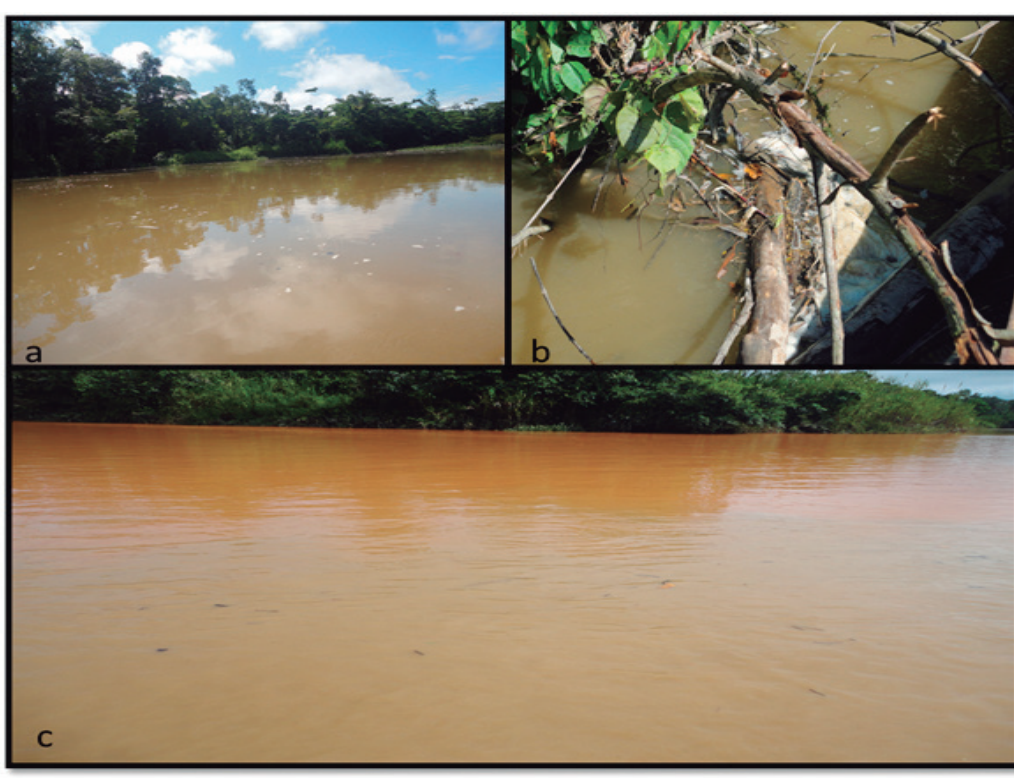

Figura 4. a, b material en suspensión en el río Sampablo. c. Turbiedad en el río Quito.

por la minería practicada directamente sobre el cauce del río, constituye una restricción en el desarrollo de la vida acuática y sus procesos biológicos, porque influye en la turbiedad del agua, quien tiene responsabilidad en fenómenos tan importantes como la fotosíntesis, la cual a su vez es responsable de la producción primaria en el ecosistema y el mantenimiento de las cadenas tróficas en el mismo, situación corroborada con Seoánez (1999), quien expresa que altos niveles de turbidez originados por materia orgánica, arcilla y material en suspensión reducen la luz en el agua, ocasionando que esta pierda la habilidad de apoyar la diversidad de organismos acuáticos.

Lo expuesto pone en evidencia las notables afectaciones del río Quito y en especial en su parte media, como consecuencia de actividades mineras desarrolladas sobre el mismo y otras fuentes hídricas que a este vierten, porque estas se ejecutan sin ningún control y sus contaminantes son vertidos sin tratamiento previo, lo contrario de un ecosistema en condiciones naturales y que no esté intervenido por actividades antrópicas, pues en estos suelen ser bajas las concentraciones de turbiedad y sólidos suspendidos. Esta situación contribuye al desarrollo óptimo de la fauna y flora presente en el ecosistema y eleva su productividad. Lo anterior se evidencia al hacer una comparación entre los datos obtenidos en esta investigación y los reportados por IIAP (2013), donde se encontraron valores de turbiedad que oscilaron entre 7 y 9,5 FAU y concentraciones de sólidos disueltos entre 20,3 y 21,9, lo que pone en evidencia la buena calidad del recurso hídrico, no solo para los organismos acuáticos sino también para las poblaciones humanas aledañas.

Por su parte, los nutrientes presentaron un comportamiento similar con tendencia a afectar el estado de salud del río como ecosistema. En la parte media de Quito, los nitratos oscilaron entre 1,52 y $23,1 \mathrm{mg} / 1$, contrario a la parte baja, donde oscilaron entre 20,05 y >35 mg/l, observándose en esta zona un incremento de las concentraciones de esta variable, indicando presencia de contaminación orgánica, que afecta el desarrollo de los organismos. Los nitritos, alcanzaron concentraciones entre $1,175 \mathrm{mg} / 1$ para la zona media y $1,05 \mathrm{mg} / \mathrm{l}$ para la baja, siendo las más elevadas en la parte media, lo que manifiesta aguas con cierto grado de contaminación orgánica 
relacionada principalmente con la existencia de asentamientos humanos representados por campamentos mineros y la comunidad en general, los cuales aportan aguas residuales y residuos sólidos domésticos que pueden incrementar este tipo de variables en el agua, a lo que se suma que todo el material orgánico contenido en el suelo es vertido directamente a la fuente durante la actividad de explotación mineral. Al respecto, Kadlec y Knight (1996), Stumm y Morgan (1970) y Martin (1995), indican que elementos como los nitritos por lo general son iones que existen de manera natural, en bajas concentraciones y no superan los $0,1 \mathrm{mg} / \mathrm{l}$, pues, de no ser así, se afecta notablemente a las comunidades biológicas que se encuentran inmersas en la fuente, lo que indica que el sistema hídrico de estudio y sobre todo su parte media, presenta concentraciones muy por encima de este valor que junto con la modificación del cauce, la pérdida de hábitat y sustratos pueden estar afectando la permanencia de especies en el área.

En cuanto al hierro, se presentaron variaciones entre 2,2, >3,30 mg/l para la parte media del río Quito y 2,7,>3,30 mg/l para la baja, situación que pudo estar influida por la remoción del sustrato durante el proceso de las actividades mineras evidenciadas durante la fase de monitoreo. Este resultado indica, que ambas zonas presentan aguas con niveles de hierro que no favorecen el desarrollo de la flora y fauna presentes en el ecosistema porque sobrepasan los valores límites permisibles por el Decreto 1594/84 (Ministerio de Agricultura 1984), el cual debe ser 0,1 mg/l, para que las especies puedan desarrollarse satisfactoriamente.

A todo lo anterior se suman las concentraciones promedios de grasas y aceites arrojadas, las cuales variaron entre 27,7 y $28,7 \mathrm{mg} / 1$, lo que evidencia aguas contaminas como resultado de los vertidos de productos oleosos, lubricantes, y combustibles de las diferentes dragas que se encuentran sobre todo en el sistema hídrico desarrollando activi- dades mineras, además de las embarcaciones que se transportan en este medio acuático y las diferentes actividades domésticas realizadas por los habitantes en la zona de influencia, afectando así la calidad del recurso a nivel estético, físico y biológico. Las concentraciones arrojadas para esta variable se encuentran por encima de los parámetros permisibles para conservación de flora y fauna que según el Decreto 1594/84 (Ministerio de Agricultura 1984), no debe superar los 0,01 $\mathrm{mg} / \mathrm{l}$, porque este tipo de materiales crean una capa sobre la superficie del agua, debido a sus características de baja densidad, poca solubilidad en agua y baja o nula biodegradabilidad, lo que hace que ocasione graves efectos en el medio acuático, relacionado con la reducción en la reoxigenación a través de la interfase aire-agua, disminuyendo el oxígeno disuelto y absorbiendo la radiación solar, afectando a la actividad fotosintéticay, en consecuencia, la producción interna en el ecosistema.

En síntesis, los resultados del estudio indican la alta influencia de la minería en algunas variables fisicoquímicas y por lo tanto en la calidad del cuerpo de agua, porque se evidencia el estado de deterioro donde se encuentra su parte media, sobre la cual son evidentes las alteraciones morfológicas y fisicoquímicas del agua que constituyen aportantes directos de contaminación al río. Al hacer una comparación por sectores como la indicada en la Figura 5, se observa que los mayores aportes de sólidos ingresan por los afluentes de la parte media, debido al aumento del material en suspensión como consecuencia de un mayor número de entables mineros en el sector y una mayor frecuencia de operación de los mismos. De ahí que las mayores concentraciones de turbiedad se evidenciaron en el mismo sector, como consecuencia de la remoción del suelo a mayor escala y destrucción de la vegetación ribereña, lo que ocasiona afectaciones en el ecosistema y reducción de su productividad. Al respecto, Álvarez et al. (2011), expresan que el incremento 


\section{Datos de Solidos y Turbiedad en la parte media del Río Quito}

\section{Datos de Solidos y Turbiedad en la parte baja del Río Quito}
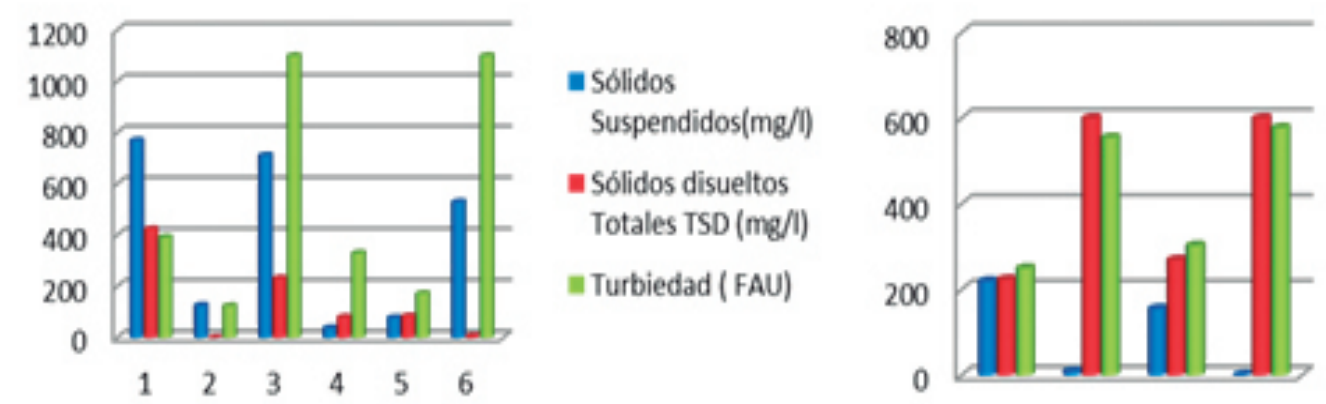

- Sólidos disueltos Totales TSD $(m g / l)$

Solidos Suspendidos $(\mathrm{mg} / \mathrm{l})$

- Turbiedad (FAU)

Figura 5. Datos de sólidos y turbiedad en la parte media y baja del río Quito.

de sólidos en suspensión en el agua por la acción de las dragas, reduce la transparencia del agua y la penetración de la luz solar, disminuyendo la tasa de fotosíntesis en el fitoplancton y de las plantas sumergidas, que son la base de la cadena trófica, y así se reduce la productividad primaria del sistema. Todo lo anterior pone en evidencia la necesidad de intervenir con estrategias de gestión, control y restauración ambiental no solo sobre el río Quito sino sobre todos sus afluentes para alcanzar resultados que sean acordes con la problemática y sostenibles en el tiempo.

Pese a que los resultados anteriores muestran un gran nivel de alteración en algunas variables fisicoquímicas de alta influencia en los procesos hidrobiológicos, como son los sólidos suspendidos y disueltos, nutrientes, hierro, grasas y aceites, variables como el oxígeno disuelto, el $\mathrm{pH}$, la temperatura entre otras, se encontraron en rangos que podrían favorecer a los organismos acuáticos y la aplicación de procesos de restauración orientados con el mejoramiento de la calidad del recurso hídrico y al restablecimiento de las condiciones naturales del ecosistema en general.

En este contexto, la temperatura y el $\mathrm{pH}$ presentaron rangos normales y pocas variaciones entre estaciones de muestreo para ambas zonas.
La temperatura, osciló entre $26,3^{\circ} \mathrm{C}$ y $27,2^{\circ} \mathrm{C}$ y el pH entre 5,2 y 6 , contexto que indica aguas cálidas correspondientes al clima de la zona geográfica de estudio y tendientes a la acidez, que contribuirían con el desarrollo de la biota acuática si se implementaran estrategias de control de contaminación, teniendo en cuenta que ambas variables son muy importantes en el equilibrio ecológico de las fuentes hídricas, por su relación de dependencia en el comportamiento de otros indicadores de la calidad del recurso como el oxígeno disuelto, los nutrientes y la conductividad. En este sentido, Seoánez (1999), manifiesta que la temperatura tiene una incidencia determinante en el comportamiento de las variables antes mencionadas, además de ser muy importante en procesos ecosistémicos debido a que tiene un efecto directo en las condiciones físicas y bilógicas del ecosistema.

En relación con el oxígeno disuelto, osciló entre $6,7,3,5,3$ y $6,2 \mathrm{mg} / \mathrm{l}$ para la parte media y baja respectivamente, mostrando en términos generales una buena disponibilidad de este elemento para grupos biológicos, teniendo en cuenta que se requieren concentraciones por encima de $4 \mathrm{mg} / \mathrm{l}$ para que un ecosistema sea favorable y pueda soportar vida acuática. Lo anterior concuerda con lo indicado por el Ministerio de Agricultura (1984), 
Malina (1996) y Mitchell et al. (1991), quienes argumentan, que aguas con concentraciones $>4$ $\mathrm{mg} / \mathrm{l}$ son benéficas para el desarrollo de la fauna y flora al interior de la fuente, de ahí que ecosistemas con altos valores de este elemento, sean una señal positiva y por lo tanto muy importantes para mantener la vida acuática en el agua.

Situación similar ocurrió con los sulfatos, los cuales se encuentran ampliamente distribuidos en la naturaleza. Sus concentraciones oscilaron entre 18 y $>80 \mathrm{mg} / 1$ en la parte media del río y 49 y $>74 \mathrm{mg} / \mathrm{l}$, para la parte baja, panorama, que pese al avanzado nivel de intervención de la cuenca como producto de las actividades mineras, no constituye un aspecto negativo porque según la OMS (2004), el sulfato solo afecta la calidad de las fuentes hídricas y la salud de las personas, cuando sus concentraciones exceden los $500 \mathrm{mg} / 1$.

Finalmente, el desarrollo actividades mineras tuvo una menor influencia en variables como la conductividad, la cual es entendida como su capacidad para transmitir corriente en relación con la presencia de iones disueltos, pues, varió entre $81,3,10,6 \mu \mathrm{s} / \mathrm{cm}$ y $15,23,19,2 \mu \mathrm{s} / \mathrm{cm}$, tanto en la zona media como baja respectivamente, lo que pudo estar relacionado con la poca presencia de material disuelto que pudiera incrementar esta variable en el agua.

En términos generales, el desarrollo de actividades mineras sobre el río Quito y otros cuerpos de agua que a este vierten, representa una alta influencia en las características fisicoquímicas del agua y por lo tanto en la calidad de la misma sobre todo en su parte media. Lo anterior, teniendo en cuenta que se incrementaron los valores de sólidos disueltos, suspendidos y totales, al igual que la turbiedad del agua y otras variables fisicoquímicas como algunos nutrientes junto con las grasas y aceites, pues los datos de estas variables, se encontraron por encima de los niveles establecidos según la norma.
A este respecto Clark (1995) expresa que el desarrollo de actividades mineras con dragas, produce una alteración del cauce de los ríos e impactos dramáticos tanto en la dinámica e hidrología de los mismos como en las comunidades bióticas asociadas. El cauce de un río es el producto de una compleja serie de factores (incluyendo las propiedades de los sedimentos, la hidráulica del curso de agua, y el transporte de sedimentos por el agua); todos ellos interactúan entre sí y cualquier alteración de uno tiene un impacto en los otros y en sus comunidades bióticas (plancton, macroinvertebrados, peces, moluscos y otros organismos acuáticos), que suelen estar adaptados a unos determinados parámetros limnológicos y de hábitat. En adición, el trabajo de campo acompañado de entrevistas y observaciones directas pusieron en evidencia el avanzado estado de deterioro de la parte media y baja del río Quito en cuanto a sus condiciones físicas, pues su cauce y riberas, se encuentran totalmente modificados (Figura 6).

Sin lugar a dudas, estas alteraciones estructurales y fisicoquímicas alteran la calidad de la cuenca, razón por la cual se puede inferir que estas alteraciones constituyen la principal causa en la baja presencia de peces como consecuencia de un desequilibrio ecológico que parte de la modificación y sedimentación del cauce, la eliminación de la vegetación de ribera que aporta nutrientes necesarios para el flujo de energía al interior del ecosistema, el aporte de grandes cantidades de sólidos y grasas, lo que puede ocasionar una reacción en cadena que afecta procesos como fotosíntesis y producción primaria, generando un mal funcionamiento fisicoquímico y biológico del sistema, lo cual contrasta con una comparación de los resultados obtenidos en este estudio con los estándares de calidad para conservación de biota acuática, la cual pone en evidencia que el ecosistema presenta condiciones fisicoquímicas que afectan el desarrollo de las especies en el interior de este. 
Bioetnia Volumen 12, 2015

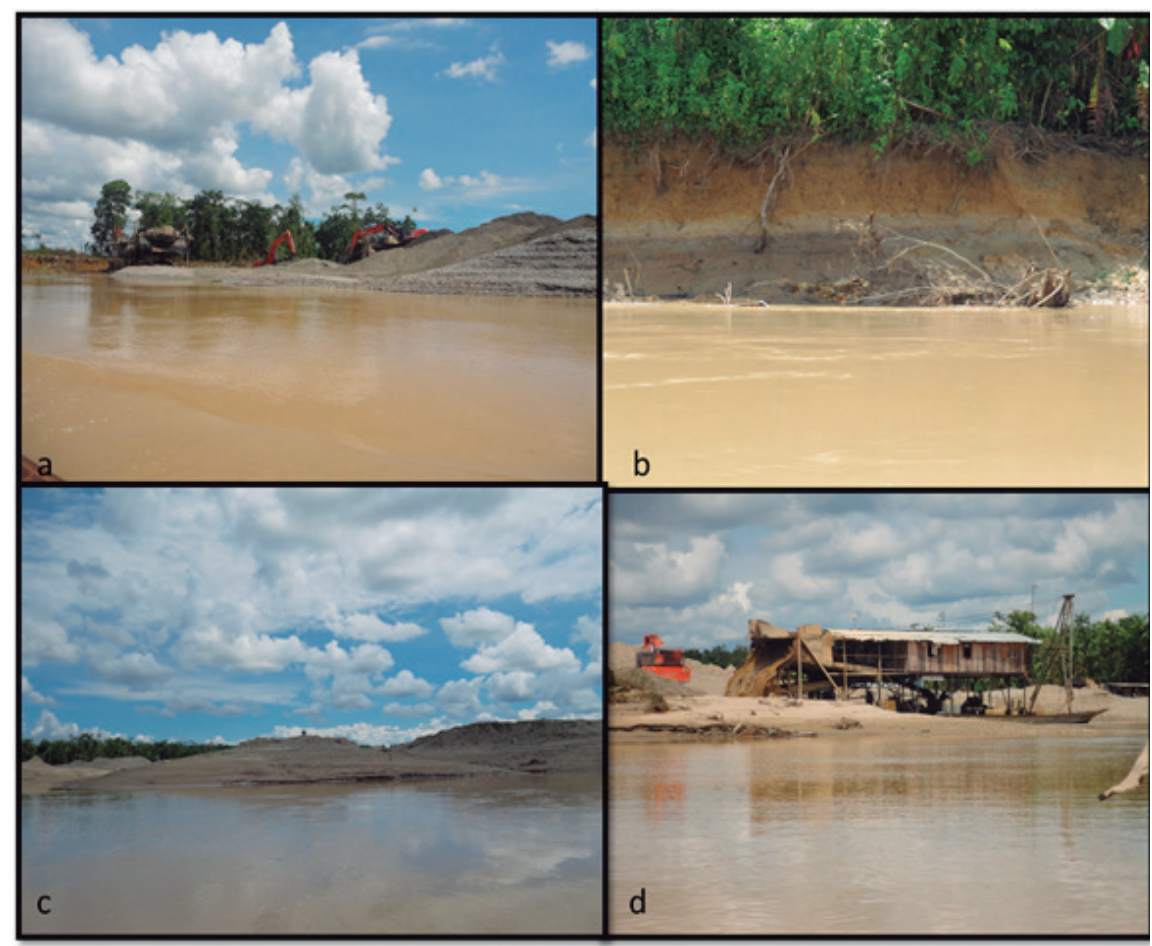

Figura 6. Modificación del cauce, turbiedad y material en suspensión presente en el río Quito.

\section{Conclusiones}

El análisis de variables fisicoquímicas evaluadas en la parte media y baja del río Quito, deja ver que esta fuente presenta condiciones que resultan restrictivo para el desarrollo de las comunidades biológicas en su interior, esto como consecuencia de la contaminación por grandes cantidades de material granulado vertido por la actividad minera practicada sin control ambiental en la zona, lo que ha ocasionado contaminación por sedimentos, afectación de las dinámicas hídricas, alteración de los sistemas de drenaje naturales y desviación del cauce y alteración de las características fisicoquímicas del agua como consecuencia del avance de esta actividad.

Se afecta todo un ecosistema del cual dependen grupos biológicos y asentamientos humanos localizados alrededor del mismo, porque el notable incremento de variables como turbiedad, sólidos, grasas y aceites, así como la modificación del cauce y la deforestación de sus riberas afecta los lugares de alimentación, refugio y reproducción de muchas especies acuáticas, incluyendo los peces, panorama que constituye un impacto tanto en los procesos ecosistémicos como en las dinámicas socioeconómicas y culturales de las comunidades negras delárea de influencia de este sistema, de ahí la importancia de diseñar e implementar medidas de control y mitigación de los impactos ocasionados por la minería sobre el medio acuático y la realización de estudios que aporten elementos para tomar decisiones adecuadas en torno a la ordenación y manejo del recurso en el territorio.

\section{Literatura citada}

Álvarez J, Sotero V, Brack A, Ipenza CA. 2011. Minería aurifera en Madre de Dios y contaminación con mercurio. Una bomba de tiempo. Lima: Instituto de la Amazonía Peruana y Ministerio del Ambiente. URL disponible en: http://biam.minam.gob.pe/novedades/ mineriamadrededios.pdf 


\section{Minería y características fisicoquímicas. Y Murillo Hinestroza, L Vargas Porras}

Clark J. 1995. Dredging can affect river flows, negatively impact wildlife, and release toxins from the sediments. Edinburgh: Earth Sciences Branch, Scottish Natural Heritage.

IIAP (Instituto de Investigaciones Ambientales del Pacífico). 2013. Caracterización ecológica de la zona alta del río Guangüi (Cauca), en comunidades indígenas de Aciesca. Chocó: IIAP; 49 pp.

Kadlec RH, Knight RL. 1996. Treatment Wetlands. In: Vymazal J (ed.). Transformactions of nutrients in natural and construted wetlands. Boca Raton: CRC Press/Lewis Publishers; 893 pp.

Malina JF Jr. 1996. Water quality. In: Mays LW (ed.). Water Resources Handbook. New York: McGraw-Hill.pp. 8.38.49. URL disponible en: https://www.amazon.com/ Water-Resources-Handbook-MAYS/dp/0070411506

Ministerio de Agricultura, Colombia. 1984. Decreto 1594 de 1984. Bogotá: MinAgricultura. 55 pp. URL disponible en: http://www.alcaldiabogota.gov.co/sisjur/normas/ Norma1.jsp? $\mathrm{i}=18617$
Millenium Ecosystems Assesment. 2005. Ecosystems and human Well-being. Synthesis. Washington, DC: World Resources Institute; 155 pp. URL disponible en: https://www.millenniumassessment.org/documents/document.356.aspx.pdf

Mitchell MK, Stapp W, Bixby K. 1991. Manual de campo de Proyecto del Río: una guía para monitorear la calidad del agua en el río Bravo. $2^{\mathrm{a}}$ ed. New Mexico: Proyecto del Río; 200 pp. Seoánez M. 1999. Ingeniería del medio ambiente aplicada al medio natural continental. $2^{\text {a }}$ ed. Madrid: Ediciones Mundi-Prensa; $710 \mathrm{pp}$. URL disponible en: https://www. agapea.com/libros/INGENIERiA-DEL-MEDIO-AMBIENTE-APLICADA-AL-MEDIO-NATURAL-CONTINENTAL-9788471147967-i.htm

OMS. 2004. Sulfate in drinking-water. Background document for development of WHO Guidelines for Drinking-water Quality. Geneva: World Health Organization; 16 pp. URL disponible en: http://www.who.int/water_sanitation_health/ dwq/chemicals/sulfate.pdf

Stumm WS, Morgan JJ. 1970. Aquatic chemistry; an introduction emphasizing chemical equilibria in natural water. New York: Wiley-Interscience; 583 pp. 